$\begin{gathered}\text { Revista do Departamento de Geografia } \\ \text { Universidade de São Paulo } \\ \text { www.revistas.usp.br/rdg }\end{gathered}$
Volume Especial - XVII SBGFA / I CNGF (2017)
ISSN 2236-2878

\title{
Aplicação do Ksn Index e do SL Index no Estudo dos Knickpoints no Perfil Longitudinal do Rio Ribeira de Iguape-SP
}

\section{Ksn Index and SL Index Application in the Study of the Knickpoints in Ribeira de Iguape River Longitudinal Profile, São Paulo State, Brazil}

\author{
André de Oliveira Souza \\ Universidade Estadual de Campinas \\ andreosouza@ige.unicamp.br \\ Archimedes Perez Filho \\ Universidade Estadual de Campinas \\ archi@ige.unicamp.br
}

\begin{abstract}
Resumo: Perfis longitudinais têm sido amplamente estudados pela ciência geomorfológica, pois se constituem como um parâmetro fundamental para a compreensão dos processos e dinâmicas fluviais. Embora os estudos por meio dessa abordagem venham sendo ampliados, muitos questionamentos têm surgido em relação à aplicabilidade desses índices em decorrência da grande complexidade dos sistemas fluviais, sobretudo àqueles de leito misto e situados em regiões tropicais úmidas. Deste modo, no trabalho apresentado o objetivo principal foi avaliar as relações existentes entre os knickpoints verificados no perfil longitudinal do rio Ribeira de Iguape, localizado no litoral sul do estado de São Paulo, com fatores litológicos, estruturais e processos autogênicos. Foram aplicados o Ksn index e o SL index que utilizam diferentes variáveis e, portanto, apresentam sensibilidades a diferentes aspectos presentes no sistema fluvial. Os resultados indicam que os processos que originaram os knickpoints apresentam grande complexidade decorrente dos múltiplos fatores ambientais que incluem a litologia, tectônica, clima (atual e subatual), além de processos autogênicos; bem como a escala utilizada no estudo. Por fim, os índices utilizados apresentaram sensibilidades diferentes às características físicas da bacia, principalmente àqueles relacionadas à declividade.
\end{abstract}

Palavras-chave: Geomorfologia Fluvial; Perfil Keywords: Fluvial Geomorphology; Longitudinal Profile; Morphometric Indexes

\begin{abstract}
Longitudinal profiles have been extensively researched by geomorphological science, since it constitutes a fundamental parameter for the understanding of fluvial processes and dynamics. Although studies using this approach have been increased, several questions have arisen regarding the applicability of these indices due to the great complexity of fluvial systems, especially those of mixed bed and located in humid tropical regions. In this paper, the main aim was to evaluate the relationship between the knickpoints found in the longitudinal profile of the Ribeira de Iguape River (São Paulo southern coast), with lithologic, structural and autogenic processes. Ksn index and SL index were used because it use different variables with sensitivities to unlike aspects of the fluvial system. The results indicate that the processes which gave origin to the knickpoints are high complex due to the multiple environmental features, which include lithology, tectonics, climate (current and past), and autogenic processes; as well as the scale used in the study. Finally, the indexes used presented different sensitivities to the physical characteristics of the basin, especially those related to slope.
\end{abstract}

Longitudinal; Índices Morfométricos 


\section{INTRODUÇÃO}

O perfil longitudinal se constitui como um importante meio de análise da evolução morfológica de canais fluviais e um dos parâmetros fundamentais para a compreensão geomorfológica de uma determinada área (LEOPOLD et al.; 1964; PHILLPS E LUTZ, 2008). De acordo com Phillips e Lutz (2008), o perfil longitudinal é amplamente utilizado nos estudos geológicos e geomorfológicos como um indicador dos estágios de evolução da paisagem, soerguimento ou subsidência tectônica, variações na resistência das litologias, mudanças no nível de base e o efeito de climas e outras mudanças ambientais.

Perfis longitudinais são obtidos a partir da correlação entre a altitude e o comprimento de um determinado canal fluvial, onde o "eixo x" corresponde ao comprimento total do canal fluvial e o "eixo y" a altitude da cabeceira até a foz. Um perfil em equilíbrio, segundo Leopold et al. (1964), Hack (1973) e Goldrick e Bishop (1995) apresenta um aspecto côncavo, enquanto que os knickpoints indicam rupturas no equilíbrio ou a manutenção do equilíbrio do canal em determinado tipo litológico (HACK, 1973, GOLDRICK e BISHOP, 1995; BISHOP et al., 2005).

Assim, de acordo com Phillips e Lutz (2008) os knickpoints, knickzones e outras convexidades são tipicamente associados a um ou mais fatores, os quais não são necessariamente independentes e mutuamente excludentes. Deste modo, é possível afirmar que a dinâmica dos perfis longitudinais está vinculada ao gradiente de declividade, características do leito fluvial, capacidade de transporte da carga sedimentar de fundo e descarga hidrológica; sendo que uma mudança em um ou mais aspectos pode conduzir a elaboração de rupturas no perfil.

Neste contexto, abordagens morfométricas têm sido comumente utilizadas nos estudos de perfis longitudinais por estarem relacionadas a um conjunto de procedimentos quantitativos cuja finalidade é mensurar formas de relevo e aspectos da rede de drenagem, possibilitando a obtenção de parâmetros para correlações espaciais entre diferentes variáveis quantitativas e qualitativas. Esse conjunto de técnicas foi bastante difundido a partir do final do século passado, como resposta ao Positivismo Lógico do Círculo de Viena, sobretudo através das escolas anglófilas e dos estudos de Horton (1945); Strahler (1957) e Schumm (1977). No Brasil, estudos iniciados por Christofoletti (1969) e Perez Filho (1978) conduziram ao desenvolvimento de pesquisas sob essa abordagem, os quais tem se ampliado como ferramentas para a investigação da evolução da paisagem, principalmente a partir da disponibilidade de Modelos Digitais de Elevação (MDEs) de alta resolução e plataformas SIGs que facilitaram os cálculos deste tipo de informação (TROIANI et al.,2014).

No presente trabalho, o perfil longitudinal do rio Ribeira de Iguape foi analisado por meio das correlações entre as formas observadas no perfil e valores resultantes da aplicação de dois índices morfométricos: Steepness index (Ksn) e Slope-Lenght index (SL index). Os dois índices são calculados utilizando diferentes variáveis, mas que possibilitam avaliar as respostas fluviais às transições litológicas, soerguimentos e subsidências, assim como aos processos autogênicos. Sendo assim, o Ksn index tem como característica principal o uso da área a montante de um trecho e a normalização das variáveis pelo índice de concavidade do canal, permitindo correlacionar taxas de soerguimento e denudação de uma determinada bacia hidrográfica com a evolução de perfis longitudinais (KIRBY E WHIPPLE, 2001, CYR et al., 2014). Por outro lado, o SL index utiliza na equação o comprimento do rio à montante de um determinado trecho, possibilitando a priori uma avaliação da influência de aspectos litológicos na conformação do perfil longitudinal (CASTILLO et al., 2014).

Contudo, é interessante observar que os índices morfométricos abordados nesse trabalho têm sido comumente utilizados em rios que apresentam o canal fluvial do tipo rochoso (bedrock). Especificamente sobre o Ksn Index, Cyr et al. (2014) e Castillo et al. (2014) destacam que uma maior eficiência nas análises por meio desse índice é obtida em canais que não se verificam heterogeneidade litológica. Por outro lado, em rios aluviais ou que apresentam características tanto de rios aluviais quanto de rios rochosos (leitos mistos), os valores de ambos os índices têm apresentado significativa disparidade, suscitando discussões sobre a aplicabilidade desses dois índices nesses tipos de sistemas, bem como a influência da pluviosidade, descarga hidrológica e interferências da maré no desenvolvimento de knickpoints.

Assim sendo, o objetivo principal desse trabalho é avaliar a influência de aspectos litológicos, estruturais e/ou autogênicos na dinâmica do perfil longitudinal do rio Ribeira de Iguape, o qual está localizado no litoral sul do estado de São Paulo. Também busca-se contribuir com as discussões sobre a aplicabilidade desses dois índices em rios que possuem canais mistos (mixed alluvial-bedrock channel) e localizados em regiões tropicais úmidas. Por fim, a hipótese principal é de que não apenas fatores litoestruturais controlam a dinâmica dos perfis longitudinais, mas também fatores associados a processos internos. 


\section{CARACTERIZAÇÃO DA ÁREA DE ESTUDOS}

A área de estudos está localizada no litoral sul dos estados de São Paulo e região nordeste do estado do Paraná. O trecho da bacia do rio Ribeira de Iguape que abrange o litoral paulista se insere no macro compartimento Brasil Tropical Atlântico de Sudeste (AB'SÁBER, 2000). Ross (2002) identificou sete macros compartimentos de relevo na bacia do rio Ribeira de Iguape, sendo quatro em terras altas e três em terras baixas. Para o autor essas unidades foram organizadas sob duas grandes morfoestruturas, denominadas por ele de Faixa de Dobramentos do Atlântico, a qual é subdividida em três Unidades Morfoesculturais: Planalto e Serra de Paranapiacaba, Serra do Mar e Morros Litorâneos, Planalto de Guapiara e Planalto do Alto RibeiraTurvo; e a unidade morfoestrutural que envolve as terras baixas, Depressão Tectônica do Baixo Ribeira, subdividida em três unidades morfoesculturais: Depressão Tectônica do Baixo Ribeira, Planície Costeira Cananéia-Iguape e Planícies e Terraços Fluviais do Baixo Ribeira.

Nesta perspectiva, a bacia estudada apresenta aproximadamente 1700 metros de amplitude altimétrica, sendo que as áreas mais elevadas estão inseridas na Faixa de Dobramentos do Atlântico; enquanto que as de menor altitude estão localizadas na Depressão Tectônica do Baixo Ribeira embutida na planície costeira (Figura 1). Sob a declividade, a bacia hidrográfica do Ribeira de Iguape apresenta de um modo geral declividades moderadas, predominando declividades entorno de 10-30\%. No baixo curso e foz do rio, as declividades são muito baixas, não ultrapassando os $10 \%$.

No âmbito geológico a bacia hidrográfica do rio Ribeira de Iguape está situada na região central da Província Mantiqueira, onde estão presentes litologias associadas à Faixa Ribeira (HEILBRON et al., 1995; HEILBRON et al., 2004; MOREIRA et al., 2007; WINTER et al., 2007). Peixoto e Theodorovictz (2009) apontam que podem ser encontrados granitos, rochas miloníticas, batólitos e xistos de idades proterozoicas, cuja gênese se associa ao Ciclo Brasiliano. Também são encontradas na área litologias mesozoicas intrusivas alcalinas (Complexo Alcalino Jacupiranga e Complexo Alcalino Juquiá). Por fim, no baixo curso são encontrados afloramentos de litologias cenozoicas relacionadas às formações Pariquera-Açu Cananéia, ambas correlatas a Sequência N50-N60 de Moreira et al. (2007).

A bacia do rio Ribeira de Iguape tem proporcionado diversos questionamentos sobre mecanismos estruturais no controle da gênese e desenvolvimento dessa porção do estado de São Paulo. Grande parte desses questionamentos se dá em virtude de diversos estudos apontarem grande espessura do pacote sedimentar, que em alguns casos chegam a ter valores acima de 100 metros de profundidade (Melo, 1990; Souza et al, 1996); por outro lado, a forma com a qual o baixo curso desse rio se apresenta assemelha-se a um golfo resultante do recuo diferencial da Serra do Mar.

Assim, trabalhos desenvolvidos nas últimas décadas têm apontado para a existência de um possível gráben ou semi-gráben nessa região, o qual foi denominado de Gráben de Iguape ou baixo gravimétrico de Iguape (SOUZA et al.,1996). Os autores afirmam a existência de anomalias gravimétricas, cuja gênese possivelmente está relacionada à reativação da Zona de Cisalhamento de Guapiara durante os eventos cenozoicos que também formaram o Rifte Continental do Sudeste do Brasil (RICCOMINI, 1989).

O lineamento Guapiara tem sido mencionado desde a década de 1970 (FERREIRA et al.1981 apud SOUZA et al.,1996 ) como um importante sistema de falhas que se estende por quase todo o território paulista seguindo a orientação NW-SE. De acordo com o trabalho mencionado, o Lineamento Guapiara é um dos principais condutos de lavas do vulcanismo mesozoico ocorrido na Bacia Sedimentar Paraná-Etendeka, sendo que na paisagem afloram altos estruturais, diques de rochas básicas e intermediárias, assim como importantes soleiras que controlam diversos sistemas fluviais. Além desse importante lineamento, também são reconhecidas na área algumas Zonas de Cisalhamentos orientadas para E-W, NE-SW e NW-SE, as quais são responsáveis pelo contato estrutural entre algumas litologias, presença de anomalias de drenagem e integram a Zona Sismogênica de Cananéia (IPT, 1984; MIOTO, 1993). 



Figura 1: Mapas de altitudes e declividades na bacia do rio Ribeira de Iguape.

A) Mapa hipsométrico da bacia do rio Ribeira de Iguape. B) Mapa de declividade da bacia do rio Ribeira de Iguape. Elaboração: Autores, 2017.

As respostas das drenagens a esses aspectos podem ser observadas em rios que apresentam padrão retilíneo com expressivas curvas abruptas no alto curso que sugerem capturas de drenagem. Neste setor, rio Ribeira de Iguape apresenta leito do tipo bedrock sendo comuns trechos sinuosos e retilíneos. No contexto do baixo curso, o canal apresenta leito do tipo aluvial, meandros bem definidos e amplas planícies de inundação. 


\section{PROCEDIMENTOS METODOLÓGICOS}

O trabalho foi desenvolvido a partir do uso do Ksn index e o SL Index, os quais foram obtidos por meio do uso de MDEs e de seus subprodutos. O SRTM (Shutter Radar Topography Mission) com 30 metros de resolução espacial utilizado nesse trabalho foi submetido a procedimentos padrões para a correção e preenchimento de pixels nulos ou que apresentavam valores negativos em topos de morros.

Deste modo, os perfis e os índices foram elaborados a partir de curvas de nível com equidistância de 50 metros, as quais foram geradas por meio do ArcGis 10.4. Especificamente, os perfis longitudinais foram plotados em uma planilha do Microsoft Excel.

\section{Ksn Index (Steepness Index)}

Esse índice morfométrico é aplicado por meio da equação $S=K s n \times A^{-\theta}$, onde o $\mathrm{S}$ corresponde ao valor da declividade, o A à área a montante do trecho analisado e o $\theta$ ao índice de concavidade. A variável correspondente à área foi obtida por meio dos valores do flow accumulation.

Em relação ao índice de concavidade (concavity index), os valores foram obtidos pela regressão linear da correlação entre a declividade e a área acumulada em log-log. Assim, obteve-se um $\theta_{\text {ref }}$ distinto e específico que foi utilizado para normalizar a variável Ksn. Geralmente, o $\theta_{\text {ref }}$ utilizado na aplicação do Steepness Index corresponde ao valor de $-0,45$, o qual é considerado pela bibliografia como referência para rios do tipo bedrock (KIRBY E WHIPPLE, 2001, CYR et al., 2014; AMBILI E NARAYAMA, 2014).

Entretanto, verificou-se que a normalização dos resultados por meio desse valor de referência conduziu a superestimação dos valores correspondentes aos knickpoints presentes nos perfis longitudinais, sugerindo que esse valor não é adequado para a análise de rios que apresentam canais fluviais mistos (mixed alluvialbedrock channel). Assim, optou-se pela normalização dos resultados com o valor do índice de concavidade do rio Ribeira de Iguape-SP.

\section{SL Index (Slope-Lenght Index)}

O índice proposto por Hack (1973) é obtido a partir da relação entre o SL index de cada trecho do rio (1) com o SL index do rio em sua totalidade (2). De acordo com Goldrick e Bishop (1995) e Castillo et al. (2014) esse índice apresenta maior sensibilidade às mudanças litológicas, enquanto que o Ksn é mais adequado para a avaliação de aspectos tectônicos pelo fato da sua normalização ser realizada pela área acumulada à montante. As equações utilizadas para o cálculo do índice são as seguintes (Equação 1 e Equação 2):

$$
\begin{aligned}
& S L=(\Delta h \div \Delta l) \times L \\
& S L=\frac{\Delta h *}{\log L}
\end{aligned}
$$

\section{Equação 1}

Equação 2

Onde: $\Delta \mathrm{h}=$ equidistância entre as curvas de nível; $\Delta \mathrm{l}=$ medida do curso em linha reta; $1=$ comprimento do trecho; $\Delta \mathrm{h}^{*}=$ Amplitude altimétrica; $\mathrm{L}=$ comprimento total do rio.

\section{RESULTADOS E DISCUSSÕES}

O perfil longitudinal do rio Ribeira de Iguape apresenta knickpoints no alto e médio curso, os quais estão localizados nos contatos entre litologias e também nos trechos influenciados pelas Zonas de Cisalhamentos Morro Agudo e Figueira, sugerindo possíveis controles litoestruturais no desenvolvimento dessas anomalias (Figura 2). Verifica-se ainda que esse setor do perfil longitudinal se encontra acima da linha de tendência, indicando a possibilidade de soerguimento dessa porção do rio ou ainda resistência litológica aos processos erosivos. Como o valor do Ksn index não demonstrou valores representativos para esse trecho, ao contrário do $S L$ index que apontou um valor significativo em congruência com a principal anomalia no perfil, é possível que a segunda hipótese seja mais bem validada. 


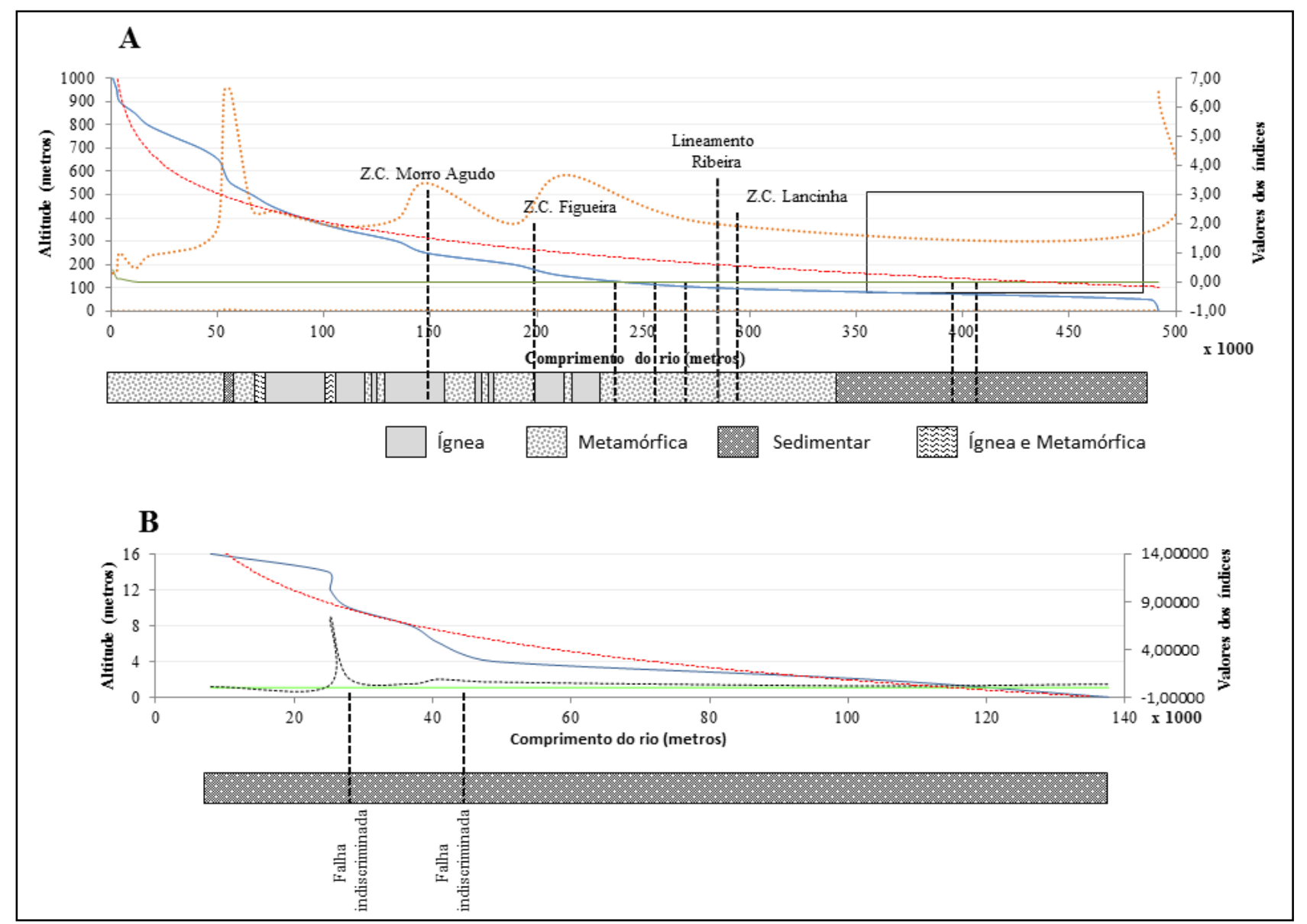

Figura 2: Perfil Longitudinal do rio Ribeira de Iguape. A) Perfil longitudinal elaborado a partir de curvas de nível com equidistância de 50m extraídas da SRTM 30m. Linha pontilhada laranja - Valores de SL index. Linha verde - valores de Ksn Index. Linha pontilhada vermelha - Linha de melhor ajuste. B) Perfil longitudinal extraído a partir de curvas com equidistância de 2 metros. Linha azul - perfil longitudinal; linha verde - valores do Ksn index; linha pontilhada preta - valores do SL index; linha tracejada vermelha - melhor ajuste do perfil. Fonte: Autores, 2017

Os valores do Ksn index próximos de zero indicaria, segundo Cyr et al. (2014) uma irrisória atividade (neo) tectônica na bacia, entretanto é provável que os valores obtidos vinculem-se ao fato de que a bacia hidrográfica do rio Ribeira de Iguape apresenta baixo gradiente de declividade (-0,507), Em contrapartida, os valores do $S L$ index apresentou maior heterogeneidade de valores e maior sensibilidade às anomalias do perfil longitudinal, sugerindo que coexistem importantes diferenças na erodibilidade das litologias (CASTILLO et al. 2014) controlando um complexo sistema de transporte sedimentar de fundo de canal e a dinâmica de elaboração dos níveis de base locais e temporários.

No baixo curso do rio Ribeira de Iguape ambos os índices não apresentaram variações significativas, inclusive no trecho onde coexistem dois falhamentos. Neste contexto, as ausências de anomalias no perfil longitudinal e nos valores dos índices indicariam a ausência de um contato entre litologias com características diferentes, assim como de uma incipiente atividade tectônica. Porém, Goldrick e Bishop (1995) e Phillips e Lutz (2008) apontam a importância da escala nos estudos sobre perfis longitudinais e, deste modo, suscita a possibilidade de que os valores inexpressivos desses dois índices, assim como da ausência de anomalias no perfil longitudinal, podem estar vinculados à questão escalar.

Deste modo, a fim de verificar a possibilidade de resposta do canal fluvial aos dois falhamentos, elaborou-se o perfil longitudinal do baixo curso com equidistância de 2 metros (Figura 2B). O resultado possibilitou o delineamento de anomalias no perfil longitudinal e nos valores do índice de Hack, embora os valores do Ksn index tenham permanecidos próximos de zero sugerindo que de fato não há evidências de soerguimentos ou subsidências no controle da rede de drenagem, além de ressaltar o fundamental papel da declividade nos valores desse índice. 
Buscando compreender a dinâmica geomorfológica por meio dos resultados desses dois índices, foi realizada a correlação matemática entre os valores dos mesmos (Figura 3A). Assim, verificou-se que o baixo valor de $\mathrm{R}^{2}(0,1298)$ e uma tendência de que essa correlação ocorre de modo negativo, sugere além de uma fraca correlação entre os valores dos índices; sob a óptica geomorfológica esses resultados sugerem que um dos aspectos investigados é mais eficiente no controle da evolução do perfil longitudinal. Como tem sido apontado, é provável que esse aspecto esteja relacionado às características litológicas e também às interferências de descarga hidrológica e oscilações de maré (baixo curso).
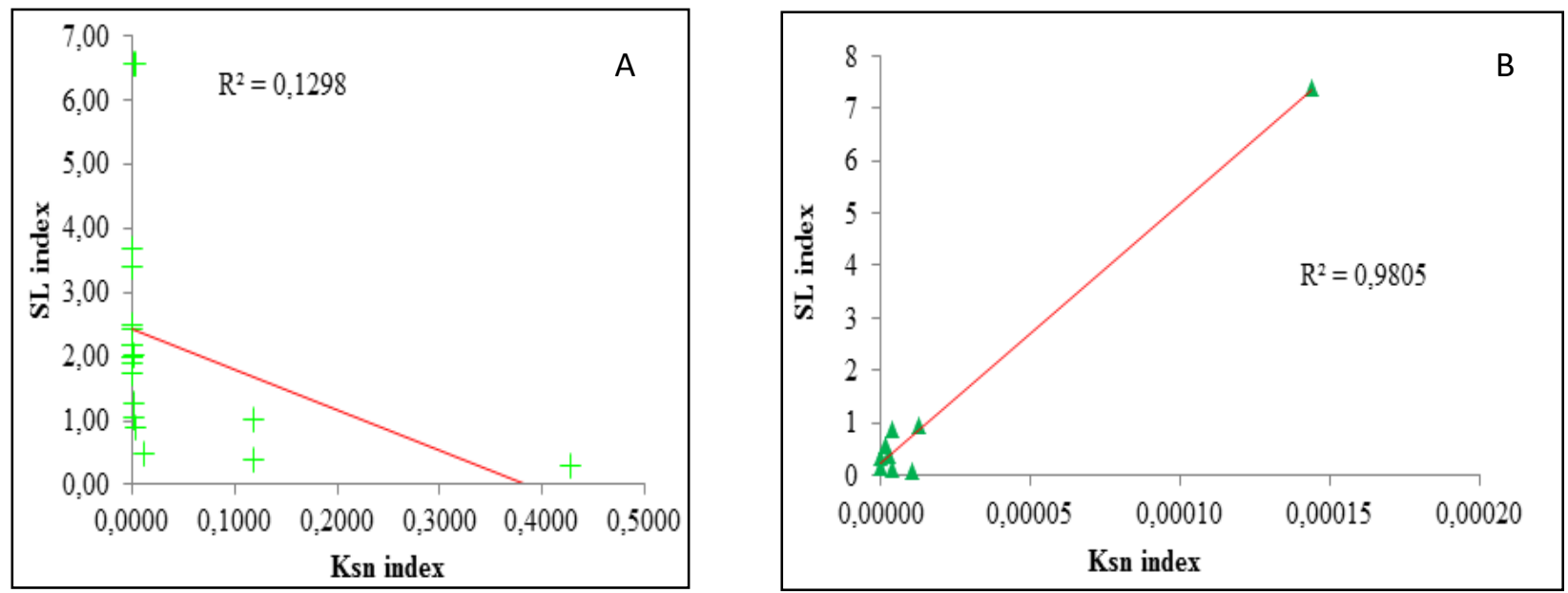

Figura 3: Gráficos das correlações lineares no Ribeira de Iguape-SP. A) Correlação entre ambos os índices no rio Ribeira de Iguape em sua totalidade. B) Correlação entre ambos os índices para o baixo curso do rio Ribeira de Iguape. Linha vermelha: regressão linear. Fonte: Autores, 2017

Sobre os aspectos hidrológicos, Flint (1974) e Phillips e Lutz (2008) pontuam a importância das oscilações na intensidade e frequência das descargas hidrológicas no desenvolvimento de knickpoints em perfis longitudinais. Embora não se tenham mensurações dos inputs vinculados a essas variáveis na dinâmica fluvial do Ribeira de Iguape, a baixa energia vinculada à topografia pouco acidentada, sobretudo a partir do médio curso favoreceu a deposição de coberturas superficiais associadas a ambientes deposicionais flúviomarinhos no médio-baixo curso, que provavelmente apresentam correlações com as oscilações de maré e episódios de transgressão e regressão marinhas Quaternárias vinculadas às mudanças na temperatura e umidade (SUGUIO et al., 1978; MELO, 1990; PESSENDA et al. 2012; SILVA, 2014). Assim, os fatores topográficos não devem exercer significativo papel erosivo, mas favorece a deposição de sedimentos e elaboração de extensas planícies aluviais e de baixos terraços.

Contudo, no baixo curso do rio Ribeira de Iguape há uma forte correlação entre ambos os índices $\left(\mathrm{R}^{2}=0,9805\right)$, indicando aparentemente a importância conjunta de aspectos litológicos e tectônicos e, portanto, no baixo curso haveria certo "equilíbrio" litoestrutural no controle dos knickpoints desse trecho do rio (Figura 3b). A existência de um gráben nessa área (SOUZA et al., 1996) explicaria as causas da dinâmica mencionada, porém é igualmente importante destacar a escala em que se é analisado esse perfil, assim como os aspectos geomorfológicos e hidrológicos da região onde a bacia se encontra; de modo que a dinâmica do canal fluvial no baixo curso provavelmente é resultante de múltiplos fatores, sugerindo que a manutenção ou não do equilíbrio fluvial está intimamente relacionada aos aspectos pluviométricos, descargas hidrológicas, litoestruturais e aos processos autogênicos de evolução fluvial (FLINT, 1974).

Deste modo, os resultados sugerem que as convexidades e rupturas presentes no Ribeira de Iguape podem estar prioritariamente associadas a controles litológicos e climáticos tendo em vista a complexidade decorrente dos inúmeros grupos de rochas pré-cambrianas, os quais são delimitados por falhamentos vinculados as principais zonas de cisalhamentos, e a alta pluviosidade na área (> $1000 \mathrm{~mm} / \mathrm{ano})$. Assim, deformações neotectônicas não exerceriam, a priori, grandes transformações na paisagem fluvial quaternária, embora a área esteja inserida em uma das áreas sísmicas mapeadas pelo (IPT, 1984). 
Por fim, as espacializações das principais rupturas no perfil longitudinal distantes da foz, sugerem duas possibilidades: (i) pode ser que recentemente não tenham ocorrido grandes ajustes na rede de drenagem, o que converge com a ideia de certa estabilidade no canal fluvial. No entanto, (ii) é possível que o Ribeira de Iguape apresente rápido ajuste às alterações nos níveis de bases principais e, assim, não possibilitaria a manutenção de convexidades significativas no perfil.

\section{CONCLUSÕES}

As respostas de ambos os índices aos knickpoints indicam que os valores do Ksn index muito baixos, resultam do baixo gradiente de declividade e sugerem que para áreas pouco declivosas ele não apresenta eficiente aplicabilidade devido o uso da área como variável normalizadora, podendo inclusive mascarar evidências de processos neotectônicos. Ao contrário, o $S L$ index, que por ser normalizado pelo Log do comprimento total do rio, apresenta maior sensibilidade às variações litológicas e permite uma melhor análise da dinâmica do canal fluvial principal de bacias com configurações topográficas pouco acidentadas, como é o caso da bacia hidrográfica do rio Ribeira de Iguape.

Por meio dos resultados também foi possível avaliar a importância da escala de análise dos perfis longitudinais, a fim de estabelecer a influência de determinados fatores no condicionamento da dinâmica fluvial, bem como no estabelecimento de estágios de equilíbrios de um sistema fluvial. No rio Ribeira de Iguape, constatou-se que a ruptura ao longo do canal fluvial é influenciada principalmente pelos contatos litológicos, à medida que diferentes tipos de rochas resultam em intensidades erosivas diferentes. Do mesmo modo, em setores onde o leito é do tipo aluvial, há a concomitância de múltiplos fatores ambientais controlando os processos de modelamento do perfil longitudinal.

Por fim, os dados indicam que os processos fluviais responsáveis pela elaboração dos perfis longitudinais em rios de leitos mistos, ou seja, que apresentam trechos do tipo bedrock e do tipo alluvial river são extremamente complexos. Assim, os valores de ambos os índices não apresentaram importante correspondência entre eles, sugerindo que em rios com essas configurações os estabelecimentos de estágios de equilíbrio são dependentes das combinações entre fatores estruturais, litológicos, climáticos e/ou processos autogênicos.

\section{AGRADECIMENTOS}

Os autores agradecem a Fundação de Amparo à Pesquisa do Estado de São Paulo (FAPESP) pelo suporte financeiro por meio do Processo Fapesp 2016/00382-9

\section{BIBLIOGRAFIA}

AB'SABER, A. N. Fundamentos da geomorfologia costeira do Brasil atlântico inter e subtropical. Revista Brasileira de Geomorfologia, UGB/UFU: Uberlândia, 1:27-43, 2000.

AMBILI, V; NARAYANA, A. C. Tectonic effects on the longitudinal profiles of the Chaliyar River and its tributaries, southwest India. Geomorphology, v. 217, p. 37-47. 2014.

BISHOP, P; HOEY, T. B.; JANSEN, J.D; ARTZA, I. L. Knickpoint recession rate and catchment area: the case of uplifted rivers in eastern Scotland. Earth Surface Processes Landform 30 (6), 767-778, 2005.

CASTILLO, M.; MUÑOZ-SALINAS, E.; FERRARI, L. Response of a landscape to tectonics using channel steepness índices (ksn) and OSL: A case of study from the Jalisco Block, Western Mexico. Geomorphology, ed. 221, p. 204-214, 2014.

CHRISTOFOLETTI, A. Análise morfométrica das bacias hidrográficas. Notícia Geomorfológica. Campinas 9 (18): 9-34, 1969.

CYR, A. J.; GRANGER, D. E; OLIVETTI, V.; MOLIN, P. Distinguishing between tectonic and lithologic controls on bedrock channel longitudinal profiles using cosmogenic 10Be erosion rates and channel steepness index. Geomorphology, 209 27-38, 2014.

FLINT, J. J. Stream gradient as a function of order, magnitude and discharge. Water Resources. Res. 10, 969973, 1974. 
GOLDRICK, G., BISHOP, P. Distinguishing the roles of lithology and uplift in the steepening of bedrock river long profiles: an example from southeastern Australia. J. Geol. 103, 227-231,1995.

HACK, J.T. Stream-profile analysis and stream-gradient index: U.S. Geological Survey. Journal Research, v. 1, n. 4, p. 421-429, 1973.

HEILBRON, M.; PEDROSA-SOARES, A. C.; CAMPOS NETO, M. C.; SILVA, L. C.; TROUW, R. A. J.; JANASI, V. A. Província Mantiqueira. Pag. 203-236. In: MANTESSO NETO, V.; BARTORELLI, A.; CARNEIRO, C. D. R.; BRITO-NEVES, B. B. (Org.). Geologia do Continente Sul-Americano: Evolução da Obra de Fernando Flávio Marques de Almeida. São Paulo: Beca, 2004. CD + 674p.

HEILBRON, M.; VALERIANO, C. M.; VALLADARES, C. \& MACHADO, N. A orogênese brasiliana no segmento central da Faixa Ribeira, Brasil. Revista Brasileira de Geociências, 25 (4): 249 - 266. 1995.

HORTON, R.E. Erosional development of streams and their drainage basins: hydrophysical approach to quantitative morphology. Geological Society of America Bulletin. 56(3):275-370, 1945.

INSTITUTO DE PESQUISAS TECNOLÓGICAS DO ESTADO DE SÃO PAULO - IPT. Análise de risco sísmico do Estado de São Paulo. Estabelecimento de medidas de proteção comunitária. (Relatório IPT, 20.573), 110 p., 1984

KIRBY, E.; WHIPPLE, K. Quantifying differential rock-uplift rates via stream profile analysis. Geology 29 (5), 415-418, 2001.

LEOPOLD, L. B., WOLMAN, M. G., MILLER, J. P. Fluvial processes in geomorphology. W. H. Freeman and Company, San Francisco, California, 544 p., 1964.

MELO, M.S. A Formação Pariquera-Açu e depósitos relacionados: sedimentação, tectônica e geomorfogênese. Dissertação (Mestrado em Geociências), Instituto de Geociências, Universidade de São Paulo (USP), 211 p. 1990.

MIOTO, J. A. Sismicidade e zonas sismogênicas do Brasil. Tese (Doutoramento em Geociências), Universidade Estadual Júlio de Mesquita (UNESP), Rio Claro, 2 volumes, 1993.

MOREIRA, J. L. P.; MADEIRA, C. V.; GIL, J. A.; MACHADO, M. A. P. Bacia de Santos. In: MILANI, E. J. (coordenador); RANGEL, H. D.; BUENO, G. V.; STICA, J. M.; WINTER, W. R.; CAIXETA, J. M.; NETO, O. C. P. Bacias Sedimentares Brasileiras: Cartas Estratigráficas. Boletim de Geociências da Petrobrás, Rio de Janeiro, v. 15, n.2, p. 531-549, 2007.

PEIXOTO, C. A. B; THEODOROVICZ, A. (2009). Geodiversidade_SP. Disponível em: IIGATESP-

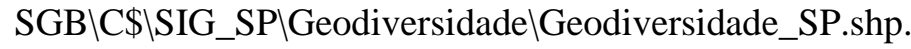

PEREZ FILHO, A. Análise estrutural da bacia hidrográfica do Rio São José dos Dourados. Dissertação (Mestrado em Geografia Física) - Faculdade de Filosofia, Letras e Ciências Humanas, Universidade de São Paulo. São Paulo, 122 p., 1978.

PESSENDA, L. C. R.; VIDOTTO, E.; DE OLIVEIRA, P. E.; BUSO-JR, A. A.; COHEN, M. C. L.; ROSSETTI, D.F.; RICARDI-BRANCO, F. Late Quaternary vegetation and coastal environmental changes at Ilha do Cardoso mangrove record, southeastern Brazil. Palaeogeography, Palaeoclimatology, Palaeoecology 363-364, 57-68, 2012.

PHILLIPS, J. D.; LUTZ, J. D. Profile convexities in bedrock and alluvial streams. Geomorphology, 102: 554566. 2008.

RICCOMINI, C. O Rift Continental do Sudeste do Brasil. Tese (Doutoramento em Geociências). Instituto de Geociências, Universidade de São Paulo (USP), São Paulo, 256p. 1989.

ROSS, J. L. S. A morfogênese da Bacia do Ribeira do Iguape e os Sistemas Ambientais. Geousp, São Paulo, v. 12, p. 21-46. 2002.

SCHUMM, S. A. The Fluvial System. Chichester: Wiley. Chichester, 338 p. 1977.

SILVA, K. C. Reconstituição paleoambiental de uma área no baixo curso do Rio Ribeira de Iguape com base em bio e geo indicadores. 2014. Dissertação (Mestrado em Geografia Física) - Faculdade de Filosofia, Letras e Ciências Humanas, Universidade de São Paulo, São Paulo, 2014. doi:10.11606/D.8.2014.tde-08052015101742. Acesso em: 2017-02-19. 
SOUZA, L. A. P. TESSLER, M. G.; GALLI, V. L. O gráben de Cananéia. Revista Brasileira de Geociências, São Paulo, v. 26, p. 139-150, 1996.

STRAHLER, A.N. Quantitative analysis of watershed geomorphology. New Halen: Transactions: American Geophysical Union, v.38. p. 913-920, 1957.

SUGUIO, K.; MARTIN, L. Formações quaternárias marinhas do litoral paulista e sulfluminense. IN: International Symposium on Coastal Evolution in the Quaternary. Special publication, n.1. São Paulo, Universidade de São Paulo, Instituto de Geociências, 1978.

TROIANI, F; DELLA SETA, M. The use of the Stream Length-Gradient index in morphotectonic analysis of small catchment: A case study from Central Italy. Geomorphology, v. 102, p. 159-168, 2008.

WINTER, W. R.; JAHNERT, R. J.; FRANÇA, A. B. Bacia de Campos. In: MILANI, E. J.; RANGEL, H. D.; BUENO, G. V.; STICA, J. M.; WINTER, W. R.; CAIXETA, J. M.; NETO, O. C. P. (coord.) Bacias Sedimentares Brasileiras: Cartas Estratigráficas. Boletim de Geociências da Petrobrás, Rio de Janeiro, v. 15, n.2, p. 511-529, 2007. 